\title{
Solvent-free model for self-assembling fluid bilayer membranes: Stabilization of the fluid phase based on broad attractive tail potentials
}

\author{
Ira R. Cooke and Markus Deserno ${ }^{\text {a) }}$ \\ Max-Planck-Institut für Polymerforschung, Ackermannweg 10, 55128 Mainz, Germany
}

(Received 8 September 2005; accepted 7 October 2005; published online 13 December 2005)

\begin{abstract}
We present a simple and highly adaptable method for simulating coarse-grained lipid membranes without explicit solvent. Lipids are represented by one head bead and two tail beads, with the interaction between tails being of key importance in stabilizing the fluid phase. Two such tail-tail potentials were tested, with the important feature in both cases being a variable range of attraction. We examined phase diagrams of this range versus temperature for both functional forms of the tail-tail attraction and found that a certain threshold attractive width was required to stabilize the fluid phase. Within the fluid-phase region we find that material properties such as area per lipid, orientational order, diffusion constant, interleaflet flip-flop rate, and bilayer stiffness all depend strongly and monotonically on the attractive width. For three particular values of the potential width we investigate the transition between gel and fluid phases via heating or cooling and find that this transition is discontinuous with considerable hysteresis. We also investigated the stretching of a bilayer to eventually form a pore and found excellent agreement with recent analytic theory.
\end{abstract}

(C) 2005 American Institute of Physics. [DOI: 10.1063/1.2135785]

\section{INTRODUCTION}

Lipid bilayers are among the most versatile of nature's biomaterials. As the interface between the cell and its environment, or between organelles and the cytosol, they provide a regulated transport of substances as small as protons to as large as entire cells. Such a wide range of functional length scales is naturally studied via an equally broad range of methods. For example, at the smallest scale detailed quantum atomistic simulations are necessary to study the transport of ions or water across the membrane interface, ${ }^{1,2}$ whereas at the opposite end of the length scale spectrum, analytic theory $^{3}$ and dynamically triangulated lattice simulations ${ }^{4}$ have been used to determine the shape behavior of whole vesicles under various conditions of pressure, volume and area, or even under hydrodynamic flow. ${ }^{5}$ Between these two extremes are problems at the so-called "mesoscale" which include membrane fusion and rupture, ${ }^{6-8}$ domain formation in multicomponent membranes, ${ }^{9-12}$ the coupling of membrane composition with curvature, or the interaction of membranes with colloidal or viral particles. ${ }^{13}$ Such problems occur at relatively large length and time scales but at the same time require a particle-based approach that reproduces the basic bilayer structure of the membrane. The combination of these two requirements necessitates the use of coarse-grained simulation approaches, in which groups of atoms are represented by single particles. Such coarse-grained approaches vary in their level of detail from a single bead per lipid ${ }^{14}$ up to quite detailed lipids on the order of ten beads. ${ }^{15}$ Naturally such a range of levels of coarse graining goes along with a trade-off between computational efficiency and level of detail. In this respect, the single most important determinant of

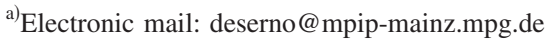

model speed appears to be the presence or absence of explicit solvent. Unlike the two-dimensional membrane, the solvent is the bulk phase which fills the entire simulation box, and accounting for its degrees of freedom can easily amount to more than $90 \%$ of simulation time. Naturally, one must include the solvent when its effects are of inherent interest to the physics of the problem. In many cases, however, its task is merely to mediate the hydrophobic attraction between lipid tails, and as such it is secondary to the overall purpose of the simulation.

If one could simulate bilayer membranes without the need for explicit solvent, a vast increase in accessible length and time scales would result, yet despite the long and successful history of solvent-free models in polymer physics, this approach has not yet been widely adopted for lipid bilayer simulations. This is because the membrane case displays one additional complication: Unlike polymers, whose structure is at the outset determined by chemistry, lipids first have to physically self-assemble into a two-dimensional fluid bilayer. This aggregation results from a balance between lipid entropy and the energy of cohesion. Since in the solvent-free case the latter stems from effective attractions (for instance, between lipid tails), a physically meaningful balance will pose restrictions on the interaction potentials. Indeed, the collective experience from the past has shown that simple choices (e.g., Lennard-Jones, LJ) do not lead to a fluid bilayer phase but only to "solid" bilayers at low temperature and low-density ("gas") phases at high temperature. Concluding that simple pair potentials are insufficient, researchers have then turned to the use of density-dependent (multibody) interactions, ${ }^{7,13,14,16,17}$ angular-dependent potentials, ${ }^{18}$ or highly tuned sets of Lennard-Jones-type potentials ${ }^{19}$ to stabilize the fluid bilayer phase without solvent (see Brannigan et al. for a recent review ${ }^{20}$ ). Unfortu- 
nately, each of these approaches suffers from one or more significant drawbacks. For example, the multibody approach introduces complications for interpretation and measurement of thermodynamic quantities, while neither the angulardependent approach nor the use of tuned LJ potentials has so far led to bilayers for which unassisted self-assembly has been demonstrated. In addition to these technical problems, it is also notable that the reported bending stiffnesses are generally outside the experimentally reported range, ${ }^{21-23}$ being restricted to either relatively low $\left(<5 k_{\mathrm{B}} T\right.$ as in Refs. 14 and 17) or high values ( $>50 k_{\mathrm{B}} T$ as in Refs. 18 and 19). Thus, there remains a clear need for an efficient solvent-free bilayer model that does not suffer from such technical drawbacks and is also highly tunable.

Recently, we have proposed a solvent-free model which sets out to solve these problems. ${ }^{24}$ This model displays a wide parameter range in which simple pair attractions drive an unassisted self-assembly into a fluid phase, within which the bending stiffness can be easily tuned to experimentally relevant values. It differs from all previous solvent-free models in its use of attractive potentials between tail beads that extend somewhat further than a simple LJ potential. As we shall show in Sec. III, our experience with broad attractions of various functional forms strongly suggests that it is this feature which ultimately enables a fluid bilayer phase to form for these strongly coarse-grained systems.

This guiding principle of broad attractions should not only apply to our model but also to solvent-free models in general. Indeed, another solvent-free model has recently been proposed by Brannigan et al. ${ }^{25}$ which is similar in spirit to ours but differs in a number of details. The principal difference is that the broad attractions in their case are restricted to special interfacial beads between the hydrophilic head and hydrophobic tail. This point, combined with a more detailed representation of lipids, is intended to capture local features of the bilayer stress profile more accurately, but at a concomitant price in efficiency. It therefore depends on the physical problem under study, as well as on ones position in the detail versus efficiency trade-off, which model is preferable in any given situation. A judicious choice then requires good knowledge of the physical properties of these models, which have so far been outlined only rather briefly, and the present paper goes toward filling this gap for the model developed by us. In Secs. II and III we briefly describe our model and discuss its self-assembly properties. In particular, we support our claim that the long-ranged nature of the attractions is the feature of key importance by showing that the qualitative physical properties are robust against the change of the specific functional form of the attraction. In Sec. IV a detailed account of the properties of the fluid phase and their variation with attractive width and temperature is given, along with a description of the gel-fluid transition. Finally, in Sec. V we study the stretching and rupture of a bilayer sheet and find near-perfect agreement with a simple theoretical model developed by Farago ${ }^{19}$ and also Tolpekina et al. $^{26}$ as well as with the experimental data.

\section{BASIC PRINCIPLES OF THE MODEL}

We describe a model surfactant system which is based on the simple idea that the solvent-mediated interaction between lipid tails can be represented by an effective attractive potential of a sufficiently broad range. Such a potential can meet the two demands of (i) providing enough cohesive energy to drive assembly of a two-dimensional aggregate, while (ii) permitting enough lateral freedom for the lipid constituents to remain in a fluid state. There are many ways in which one might construct a coarse-grained model based around the central principle of such a broad attractive potential. Below, we shall present two alternatives which differ in the form of their tail attractions. In showing both of these we merely seek to demonstrate that it is not the precise functional form that matters but the attractive range.

In this paper we present the most coarse-grained version of our model, because this is the most useful in terms of length scales obtainable. Nonetheless, it is worth noting that the same principles can trivially be extended to include more detailed lipids with greater numbers of atoms should this be required. Of course, one should always remember that by increasing the number of lipid beads, the ratio of simulation lengths to real lengths becomes less favorable. The lipids we use are represented by one "head" bead followed by two "tail" beads. Their size is fixed via a Weeks-ChandlerAndersen potential,

$$
V_{\text {rep }}(r ; b)=\left\{\begin{array}{cc}
4 \epsilon\left[(b / r)^{12}-(b / r)^{6}+\frac{1}{4}\right], & r \leqslant r_{c} \\
0, & r>r_{c},
\end{array}\right.
$$

with $r_{c}=2^{1 / 6} b$. We use $\epsilon$ as our unit of energy. In order to ensure an effective cylindrical lipid shape we choose $b_{\text {head,head }}=b_{\text {head,tail }}=0.95 \sigma$ and $b_{\text {tail,tail }}=\sigma$, where $\sigma$ is our unit of length. The three beads are linked by two finite extensible nonlinear elastic (FENE) bonds,

$$
V_{\text {bond }}(r)=-\frac{1}{2} k_{\text {bond }} r_{\infty}^{2} \log \left[1-\left(r / r_{\infty}\right)^{2}\right] \text {, }
$$

with stiffness $k_{\mathrm{bond}}=30 \epsilon / \sigma^{2}$ and divergence length $r_{\infty}=1.5 \sigma$. Lipids are straightened by a harmonic spring with rest length $4 \sigma$ between head bead and second tail bead,

$$
V_{\text {bend }}(r)=\frac{1}{2} k_{\text {bend }}(r-4 \sigma)^{2},
$$

which corresponds in lowest order to a harmonic bending potential $\frac{1}{2} k_{\text {bend }} \sigma^{2} \vartheta^{2}$ for the angle $\pi-\vartheta$ between the three beads. We fixed the bending stiffness at $k_{\text {bend }}=10 \epsilon / \sigma^{2}$.

The absence of explicit solvent molecules and the hydrophobic effect they would give rise to are compensated by an attractive interaction between all tail beads. We compared two alternative potentials that account for this effect (see insets to Figs. 1 and 2). The first of these,

$$
V_{\cos }(r)=\left\{\begin{array}{cc}
-\epsilon, & r<r_{c} \\
-\epsilon \cos ^{2}\left[\pi\left(r-r_{c}\right) / 2 w_{c}\right], & r_{c} \leqslant r \leqslant r_{c}+w_{c} \\
0, & r>r_{c}+w_{c},
\end{array}\right.
$$

describes an attractive potential with depth $\epsilon$ which for $r>r_{c}$ smoothly tapers to zero. In this case, tuning the decay 


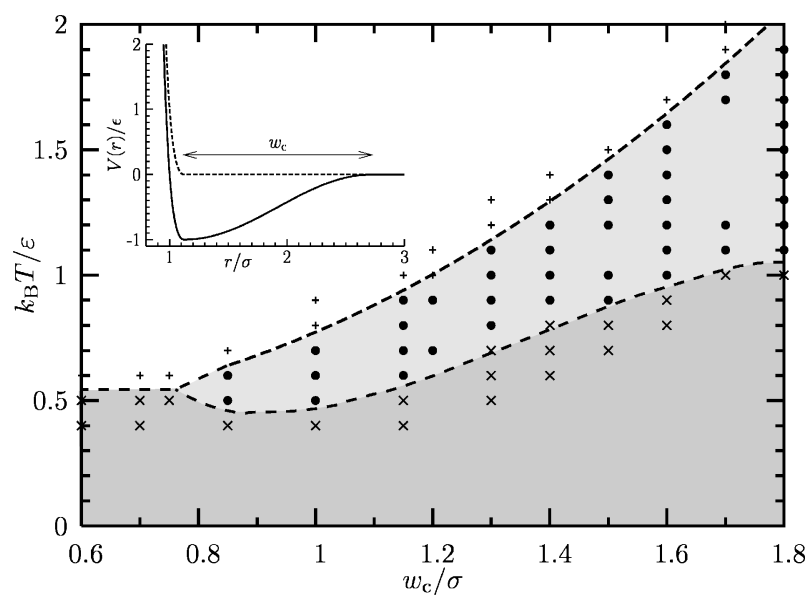

FIG. 1. Phase diagram resulting from $V_{\text {cos }}$ cohesion [Eq. (4)] in the plane of potential width $w_{c}$ and temperature at zero lateral tension. Each symbol corresponds to one simulation and identifies different bilayer phases: $(\times)$ gel, (@) fluid, and (+) unstable. The lines are merely guides to the eye. The inset shows the pair potential between tail lipids (solid line) and the purely repulsive head-head and head-tail interactions (dashed line).

range $w_{c}$ proves to be the key to obtaining a fluid bilayer state.

The second alternative is based on the familiar LennardJones potential but extends its range simply by inserting a flat piece of length $w_{f}$ at the minimum,

$$
\begin{aligned}
& V_{\text {flat LJ }}(r) \\
& =\left\{\begin{array}{cc}
-\epsilon, & r<r_{c}+w_{f} \\
4 \epsilon\left\{\left[b /\left(r-w_{f}\right)\right]^{12}-\left[b /\left(r-w_{f}\right)\right]^{6}\right\}, & r_{c} \leqslant r \leqslant w_{f}+w_{\text {cut }} \\
0, & r>w_{f}+w_{\text {cut }},
\end{array}\right.
\end{aligned}
$$

where our key tuning parameter is now the width $w_{f}$ of the flat region. The potential is cut off beyond $w_{f}+w_{\text {cut }}$, where $w_{\text {cut }}=2.5 \sigma$ is set to the usual value. Note that tuning $w_{f}$ achieves a broad potential $V_{\text {flat LJ }}$ simply by introducing a flat region, whereas in the case of $V_{\text {cos }}$ the tuning parameter $w_{c}$ varies decay range and shape simultaneously.

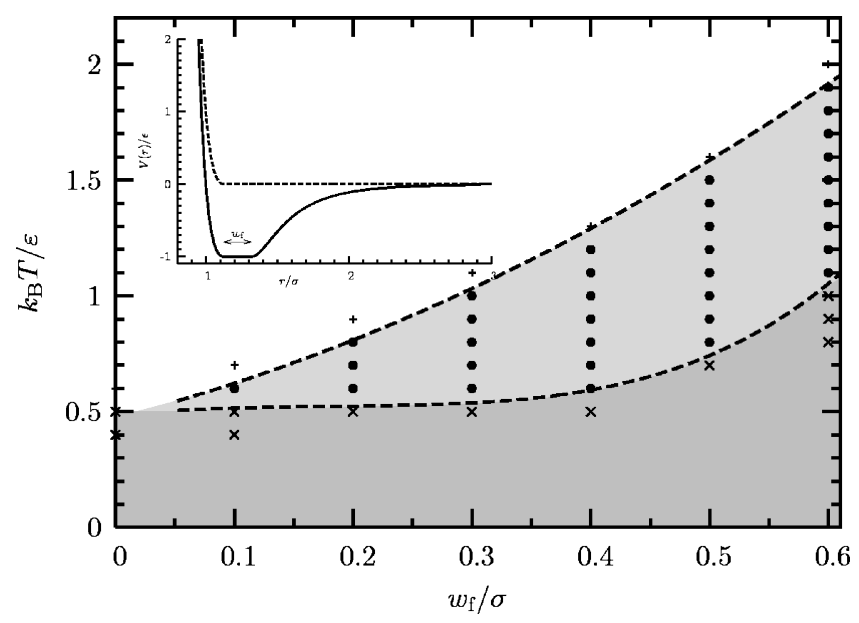

FIG. 2. Phase diagram resulting from $V_{\text {flat LJ }}$ cohesion [Eq. (5)] in the plane of potential width $w_{f}$ and temperature at zero lateral tension. The meaning of all symbols is the same as in Fig. 1.
The above model is sufficiently simple to allow implementation with a variety of molecular-dynamics (MD) or Monte Carlo integration schemes. In this work we performed MD simulations with a Langevin thermostat to obtain the canonical ensemble ${ }^{27}$ (time step $\delta t=0.01 \tau$ and a friction constant $\Gamma=\tau^{-1}$ in Lennard-Jones units ${ }^{28}$ ). Constant volume simulations were performed using a cuboid box with sides $L_{x}=L_{y}, L_{z}$ subject to periodic boundary conditions. If needed, constant tension conditions were also implemented via a modified Andersen barostat ${ }^{29}$ allowing box resizing in $x$ and $y$ dimensions only (with a box friction $\Gamma_{\text {box }}=2 \times 10^{-4} \tau^{-1}$ and box mass within the range $Q=10^{-5}-10^{-4}$ ).

All simulations were performed using the ESPRESSO program. ${ }^{30}$ Lipid membrane specific analysis and setup was done using the MBTOOLS package which is included as part of the main ESPRESSO distribution.

\section{BILAYER STABILITY AND SELF-ASSEMBLY}

In this section we shall map out the conditions under which a tensionless fluid bilayer state is stable. To do this we identified the fluid phase in two different ways (see Figs. 1 and 2 for the following). In the first method, a box-spanning bilayer was preassembled from 1000 lipids, and its equilibration under zero lateral tension was attempted. This resulted in one of three possible outcomes: a stable fluid bilayer, a gel phase with strongly increased lipid order and much lower diffusion constant, or complete breakup of the system to form a gas phase. The transition between gel and liquid occurred over a narrow temperature range and will be explored in more detail in Sec. IV C. The gas phase was always identified as the point at which the imposition of zero tension conditions resulted in a divergence in the box length.

Our second method for identifying the fluid bilayer phase was designed to ensure that we were not artificially stabilizing the bilayer due to preassembly. In this case we conducted constant volume simulations starting from a random gas configuration. Under all conditions which previously gave stable tensionless membranes, a bilayer patch quickly self-assembled which, at the correct box size, could zip up with its open ends to span the box (see Fig. 3 for such a sequence). If the box was too big, the patch either remained free or (sometimes) closed upon itself to form a vesicle. For rather large values of the width parameter $w_{c}$ or $w_{f}$ the line between fluid bilayer and the isotropic gas state becomes less distinct. For example, we observed that for $w_{c} \gtrsim 1.6 \sigma$ and $w_{f} \gtrsim 0.4 \sigma$, self-assembly to box-spanning bilayers (at constant volume) could occur well above the evaporation boundary and that immediately below this boundary we observed rather indistinct bilayers, with particularly high flip-flop rates and low orientational order. Although we will see in Sec. IV A 2 that these relatively disordered states still show a definite bilayer structure, we should not be surprised if strange behaviors are found in this small region, and we would therefore caution against the physical interpretation of such results without additional checks for model artifacts.

Using the methods mentioned above, we determined the phase diagrams for both types of attractive tail potentials, Eqs. (4) and (5), shown in Figs. 1 and 2, respectively. The 

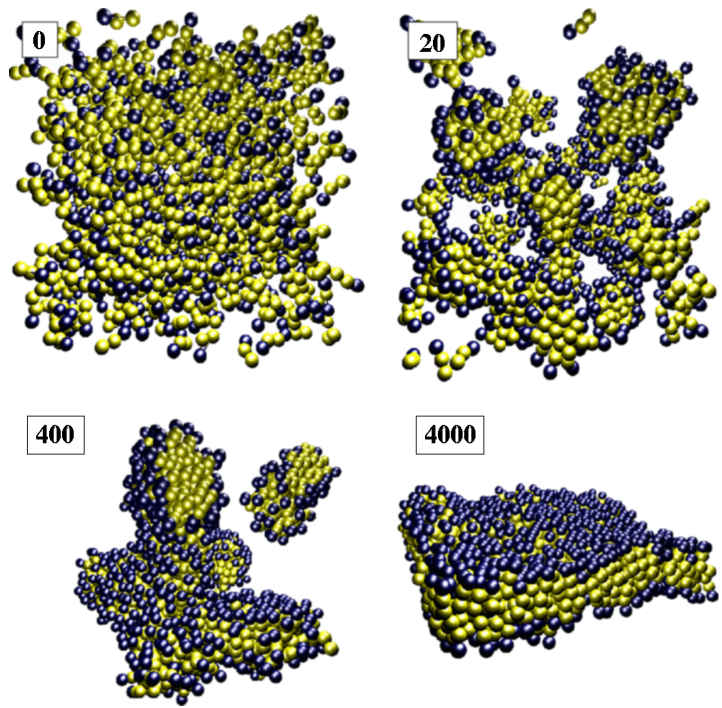

FIG. 3. Self-assembly sequence for the bilayer system with 1000 lipids in a cubic box of side length $25 \sigma$. Lipid cohesion was set to $w_{c} / \sigma=1.4\left(V_{\cos }\right)$ and temperature to $k_{\mathrm{B}} T=\epsilon$. A random gas of lipids quickly forms small clusters which slowly coarsen and eventually "zip up" to form a box-spanning bilayer sheet. The numbers indicate the MD time.

most important point to note is that in both cases we see a fluid bilayer region that broadens significantly as the potential width is increased. In this sense, both phase diagrams are remarkably similar given the strong differences in the nature of the functional forms and tuning parameters used to obtain them. At a more detailed level, one can see differences in the shape of the transition lines between the two models. This is likely due to the fact that the two width parameters work entirely differently. In the case of the cosine attraction, Eq. (4), the attractive gradient is actually varied along with $w_{c}$, whereas for Eq. (5), varying $w_{f}$ leaves the attractive shape unchanged and merely adds a region of zero force close to the particle.

Based on the relative similarity of our results for two such radically different potentials, our expectation is therefore that other potentials with a broad attractive width should also share the important property of exhibiting a stable fluid bilayer phase. This finds further support in the observation that the recent model of Brannigan et al. ${ }^{25}$ also uses a broad $1 / r^{2}$ attraction. Of course, a direct comparison is difficult because they restrict this attraction to the interface bead between the lipid head and tail. Notwithstanding the physical motivation of this choice as being in accord with the knowledge of the lateral stress profile, it would be interesting to check whether fluidity is ultimately insensitive to this detail and rests on the long range alone.

Faced with these possibilities, as well as others we might think of for the tail attraction, the question is now which to choose. Since our potential attempts to capture the effects of solvent exclusion, lipid-lipid interactions, and the fact that our three-bead "lipid" is a highly coarse-grained representation of the real thing, it is difficult to guess what its functional form should look like. Instead we could attempt to differentiate between models on the basis of their emergent physical properties; however, it turns out that both are highly tunable over a similar range. Therefore our primary consid- erations are practical ones. In this regard the cosine attraction, Eq. (4), is preferable since it acts over a slightly shorter range than the broadened $\mathrm{LJ}$ potential and is therefore faster to compute. The remainder of this paper will therefore focus on it alone.

\section{PROPERTIES OF THE FLUID PHASE}

We have characterized the bilayers in both fluid and gel phases by several observables, including the lipid orientational order parameter, cross-bilayer density profiles, and bending modulus, as well as the dynamical quantities diffusion constant and flip-flop rate. In Sec. IV A we shall define these quantities and explain in detail how they are measured. In Sec. IV B the results are presented as cross sections at constant temperature, while Sec. IV C examines the fluid-gel transition via cross sections at constant potential width.

\section{A. Observables}

\section{Orientational order parameter}

Lipids in the fluid $L_{\alpha}$ phase are on average oriented parallel to the bilayer normal. The amount of alignment can be quantified by an orientational order parameter $S$, defined by

$$
S=\frac{1}{2}\left\langle 3\left(\mathbf{a}_{i} \cdot \mathbf{n}\right)^{2}-1\right\rangle_{i},
$$

where $\mathbf{a}_{i}$ is the unit vector along the axis of the $i$ th lipid, $\mathbf{n}$ is the average bilayer normal, and the angular brackets indicate an average over all lipids. A completely isotropic system will have $S=0$ whereas a fully ordered crystalline bilayer will have $S=1$.

\section{Cross-bilayer density profile}

In a well-defined bilayer the lipid distribution perpendicular to the bilayer plane is very regular. In particular, each of the constituent beads should occupy a well-defined vertical distance from the bilayer midplane. To investigate this aspect of fluid bilayer structure we have calculated the number density of beads $\rho(z)$ as a function of vertical distance $z$ from the local bilayer midplane. We used systems with 4000 lipids at constant zero tension with a lateral box size of $L_{x}=L_{y} \approx 50 \sigma$. Although such a large system provides good statistics, it also introduces the problem of dealing with undulations. We solved this by first assigning lipids to a 16 $\times 16$ grid in the $x y$ plane and measuring the height $z$ with respect to the average local height for that grid cell. Failure to do so will overestimate the width of the distributions significantly.

Calculations of $\rho(z)$ for weakly coarse grained ${ }^{31}$ or fully atomistic lipid bilayers ${ }^{32}$ typically show differences between the shape and width of $\rho(z)$ for each functional group in the lipid. Of course, a strongly coarse-grained model such as ours cannot hope to reproduce such subtleties. Our main concern is rather to ensure that the bilayer is not merely a loose agglomeration of lipids but that these lipids are oriented approximately vertically and that they do not strongly interdigitate (although such interdigitated phases do occur naturally under certain circumstances, ${ }^{33,34}$ they are not the "norm" for a fluid bilayer). From Fig. 4 one can verify that a welldefined bilayer structure is indeed present for our system. 


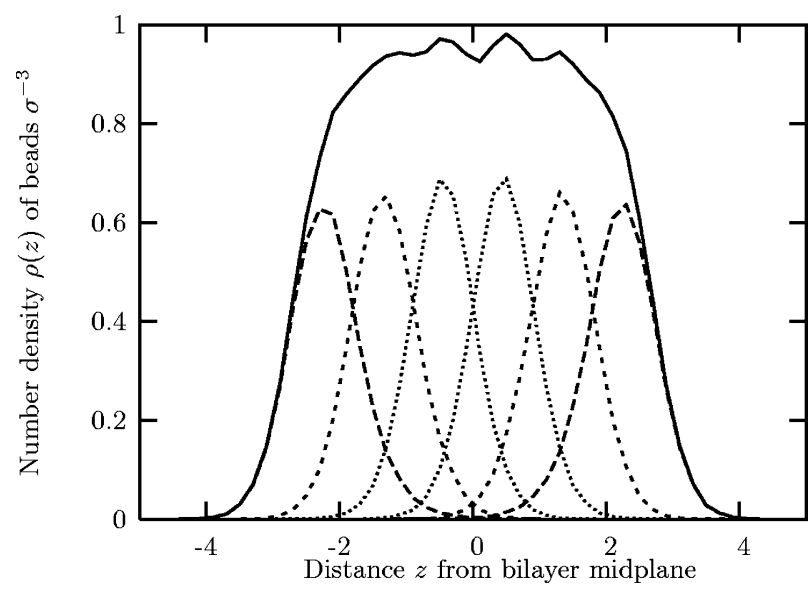

FIG. 4. Profile of the density $\rho$ as a function of vertical distance $z$ from the bilayer midplane for a system of 4000 lipids at constant zero tension and with simulation parameters $k_{\mathrm{B}} T=1.1 \epsilon$ and $w_{c}=1.6 \sigma$. The plotted lines are bead densities for head beads (long dashed), first tail beads (short dashed), terminal tail beads (dotted), and the sum of all beads (solid).

Each of the three-lipid beads shows a sharp peak about its average $z$. While the width of these peaks broadens upon approaching the liquid-gas boundary, their location is relatively stable. The upper and lower head beads are separated by a distance of approximately $4.5 \sigma$, while the inflection points of the summed density are separated by about $5 \sigma$. This agrees with our expectations for vertically oriented lipids having only minor interdigitation. Further evidence for distinct bilayer leaflets can be seen in the summed bead density which shows a slight peak for each of the terminal tail beads and a minimum at the bilayer midplane.

In order to quantitatively examine the trends in peak broadening and overlap throughout the fluid-phase region of our phase diagram we define an overlap parameter $\Psi$ as follows:

$$
\Psi=\frac{1}{\Omega} \sum_{i<j} \int d z \rho_{i}(z) \rho_{j}(z),
$$

where $i$ and $j$ are labels for the bead type (i.e., head, tail ${ }_{1}$, and tail ${ }_{2}$ ) and $\Omega=\Sigma_{i} \int d z\left(\rho_{i}(z)\right)^{2}$ is a normalization factor. From this definition we see that a complete overlap (or bead equivalence) is indicated by $\Psi=1$, whereas a rigid crystalline structure with no overlap would give $\Psi=0$.

\section{Bending modulus}

One of the key material properties of a macroscopic membrane is its bending stiffness, which measures the energetic cost per unit area of imposing a local curvature. More precisely, the classical continuum description ${ }^{35,36}$ in the limit of almost flat membranes states that the energy of a deformed piece of membrane is given by

$$
E=\frac{1}{2} \int d x d y\left[\kappa(\Delta h)^{2}+\Sigma(\nabla h)^{2}\right],
$$

where $\kappa$ and $\Sigma$ are the bending modulus and lateral tension, respectively, and where $h(x, y)$ describes the height of the membrane above some reference plane ("Monge gauge"). If we expand $h(x, y)$ in Fourier modes according to

$$
h(\mathbf{r})=\sum_{\boldsymbol{q}} h_{\boldsymbol{q}} e^{i \boldsymbol{q} \cdot \mathbf{r}} \quad \text { with } \mathbf{q}=\frac{2 \pi}{L}\left(n_{x}, n_{y}\right)
$$

and insert the result into Eq. (8), we see that the energy reduces to a sum of uncoupled harmonic oscillators with the modes $h_{\boldsymbol{q}}$ as the degrees of freedom. From the equipartition theorem we then get the power spectrum of modes as ${ }^{3}$

$$
\left\langle\left|h_{q}^{2}\right|\right\rangle=\frac{k_{\mathrm{B}} T}{L^{2}\left[\kappa q^{4}+\Sigma q^{2}\right]} .
$$

It is standard practice to obtain the bending modulus from a fit of the measured fluctuation spectrum to Eq. (10). However, some care has to be taken here. First, Eq. (8) is a continuum description and thus requires us to focus on large length scales. But for wave vectors $q$ smaller than $q_{c}$ $=\sqrt{\Sigma / \kappa}$ the dominant influence is the tension $\Sigma$, and $\left\langle\left|h_{q}^{2}\right|\right\rangle$ $\sim q^{-2}$ is insensitive to $\kappa$, so larger wave vectors than $q_{c}$ are needed. However, once $1 / q$ becomes comparable to the bilayer thickness, simple continuum theory breaks down and further effects (e.g, protrusion modes ${ }^{37}$ ) set in. Identifying the characteristic $q^{-4}$ scaling of the bending regime over a sufficiently wide range thus requires $q_{c}$-and therefore the lateral tension $\Sigma$ - to be as small as possible. Unfortunately, it turns out to be extremely hard to eliminate any remaining tension by adjusting the simulation box size by hand, since the tension depends very sensitively on the bilayer area (see Sec. V below). We avoided this difficulty by instead using a modified Andersen barostat ${ }^{29}$ to simulate in an ensemble of constant zero tension. Furthermore, to get away from microscopic lengths we took systems four times as big as the ones we used for mapping the phase diagram (4000 lipids, $L \simeq 50 \sigma$ ). Note that reaching the continuum limit in MD simulations is not trivial, since the relaxation time of bending modes scales as $q^{-4}$.

After setting up the bilayer, we first waited until the tension, box length, and energy had equilibrated (which took typically $10^{4} \tau$ for fluid systems). Then on the order of 100 configurations separated by $500 \tau$ were used to measure the mode spectrum. The bilayer midplane was identified by tracking the tail beads and interpolating their vertical position onto a $16 \times 16$ grid. Possible stray lipids had to be excluded from this procedure. A fast Fourier transform then yields the power spectrum $\left\langle\left|h_{q}^{2}\right|\right\rangle$, but this requires one important additional correction due to amplitude undersampling on a grid which we briefly discuss in Appendix A. Figure 5 provides a typical example of such a mode spectrum from which we can clearly see the asymptotic $q^{-4}$ scaling, but also the deviations at large $q$. In this case length scales exceeding $L \approx 20 \sigma$ (i.e., about four times the bilayer thickness) are required to reach the asymptotic regime. Hence, a simulation of smaller systems (1000 lipids, $L \approx 25 \sigma$ ) would not suffice to obtain a fluctuation spectrum which could in any meaningful way be fitted to Eq. (10). 


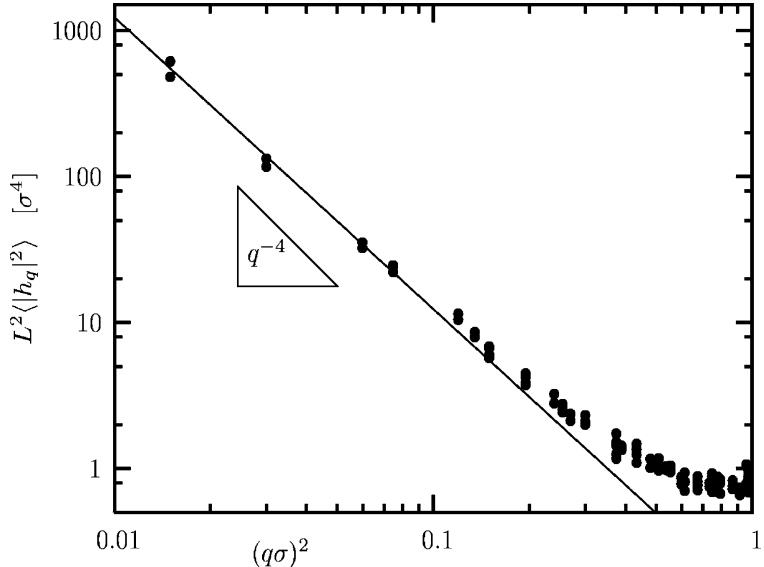

FIG. 5. Asymptotic $q^{-4}$ scaling of the power spectrum $\left\langle\left|h_{q}^{2}\right|\right\rangle$ for the bilayer system with $w_{c} / \sigma=1.4$ and $k_{\mathrm{B}} T / \epsilon=1.0$.

\section{Diffusion constant}

Calculating the in-plane diffusion constant $D_{b}$ for lipids in a solvent-free bilayer system is not as trivial a task as it might at first seem. For a strictly two-dimensional diffusive system we would of course have

$$
D_{b}=\frac{1}{4 \Delta t}\left\langle\left[\mathbf{r}_{\|, i}(t+\Delta t)-\mathbf{r}_{\|, i}(t)\right]^{2}\right\rangle_{i},
$$

where $\mathbf{r}_{\|, i}(t)$ is the projection ${ }^{38}$ of the position vector of the $i$ th lipid at time $t$ into the bilayer plane, $\Delta t$ is the time difference over which diffusion is probed, and the angular brackets indicate an average over all $N$ lipids as well as configurations separated by a time difference of $\Delta t$. The difficulty, though, is that the system is really three dimensional and that a small but nonvanishing fraction $\alpha \ll 1$ of all lipids (typically $\alpha \approx 1 \%-2 \%$ ) resides in the gas phase surrounding the bilayer. Even though they are the minority phase, they of course diffuse much faster and might thus contribute significantly to the average mean-squared horizontal distance traveled. Unfortunately it is not straightforward to eliminate such stray lipids from the average $\langle\cdots\rangle_{i}$ over all lipids in Eq. (11), since this would require us to check on the positions of all lipids during all times between $t$ and $t+\Delta t$-information that is not necessarily available.

Fortunately there is a way to check Eq. (11) which does not require us to follow lipids, namely, by looking at the entire distribution function $P(s)$ of (squared) lipid displacements $s=\left(\Delta \mathbf{r}_{\|}\right)^{2}$. If we think of lateral lipid diffusion as being due to two independent simple diffusion processes-one with bilayer diffusion constant $D_{b}$ and one with an effective lateral diffusion constant $D_{g} \gg D_{b}$ through the gas phase-a simple calculation would suggest

$$
P(s, \Delta t)=(1-\alpha) \frac{e^{-s / 4 D_{b} \Delta t}}{4 D_{b} \Delta t}+\alpha \frac{e^{-s / 4 D_{g} \Delta t}}{4 D_{g} \Delta t} .
$$

Indeed, a histogram of squared traveled distances shows exactly this double exponential decay, with the short-distance behavior dictated by the real bilayer diffusion constant $D_{b}$ (see Fig. 6). We tested in different cases (including ones with a rather large fraction of stray lipids) that $D_{b}$ is independent of $\Delta t$ and, in fact, given by the diffusion constant obtained

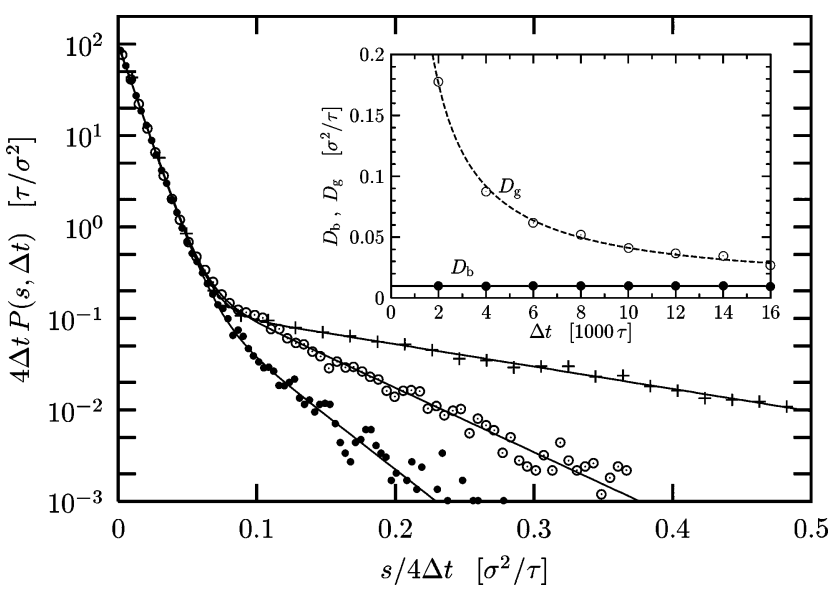

FIG. 6. Scaled distribution of squared displacements $s$ of lipid molecules for the system with $w_{c} / \sigma=1.6, k_{\mathrm{B}} T / \epsilon=1.1$ and $\Delta t=2000 \tau$ (crosses), $\Delta t$ $=6000 \tau$ (open circles), and $\Delta t=12000 \tau$ (filled circles). The lines are fits to Eq. (12). These always yield $\alpha \approx 0.02$ and values of the two diffusion constants as given in the inset. While $D_{b}$ is constant, the data are compatible with $D_{g}$ approaching $D_{b}$ with a $1 / \Delta t$ asymptotics (inset, dashed line).

from the much simpler analysis via Eq. (11). In contrast, $D_{g}$ depends essentially inversely on $\Delta t$, which rather than suggesting that diffusion through the gas phase somehow slows down reminds us that free diffusion through three space is ultimately cut short via readsorption in the bilayer or its periodic image.

Having thus established that fast moving stray lipids have no significant influence on the bilayer diffusion constant as measured via Eq. (11), we subsequently used this simpler analysis to obtain $D_{b}$. In order to make best possible use of our data we calculated $D_{b}$ by taking the average over all possible values of the starting time $t$ for each interval length $\Delta t$ [which varied from $2000 \tau$ up to the entire length of an equilibrium simulation run $(\sim 100000 \tau)]$ and then took the weighted average over all possible values of $\Delta t$.

\section{Flip-flop rate}

A lipid molecule will not stay forever in the particular monolayer in which it presently resides; rather, there is a particular probability per unit time, the flip-flop rate $r$, that it changes the monolayer. Let $N_{+}(t)$ and $N_{-}(t)$ be the number of lipids in the upper or lower monolayer at time $t$, which were present in the upper layer at time $t=0$. These numbers satisfy the master equations,

$$
N_{ \pm}(t+d t)=N_{ \pm}(t)(1-r d t)+r d t N_{\mp}(t) .
$$

The total number of such lipids is conserved, $N_{+}(t)+N_{-}(t)$ $=N / 2$, so we obtain $d N_{ \pm} / d t=-r\left[2 N_{ \pm}-N / 2\right]$. The solution satisfying the initial conditions $N_{+}(0)=N / 2$ and $N_{-}(0)=0$ is $N_{ \pm}(t)=\frac{1}{4} N\left(1 \pm e^{-2 r t}\right)$. The same considerations hold for lipids which at $t=0$ were in the lower monolayer. Hence, the total fraction $f(t)$ of lipids which at time $t$ resides in the same monolayer as they did at time $t=0$ is given by

$$
f(t)=\frac{1}{2}\left(1+e^{-2 r t}\right) .
$$

This fraction is easily measurable in simulations. A fit to Eq. (14) then yields the flip-flop rate $r$. Notice that the probabil- 

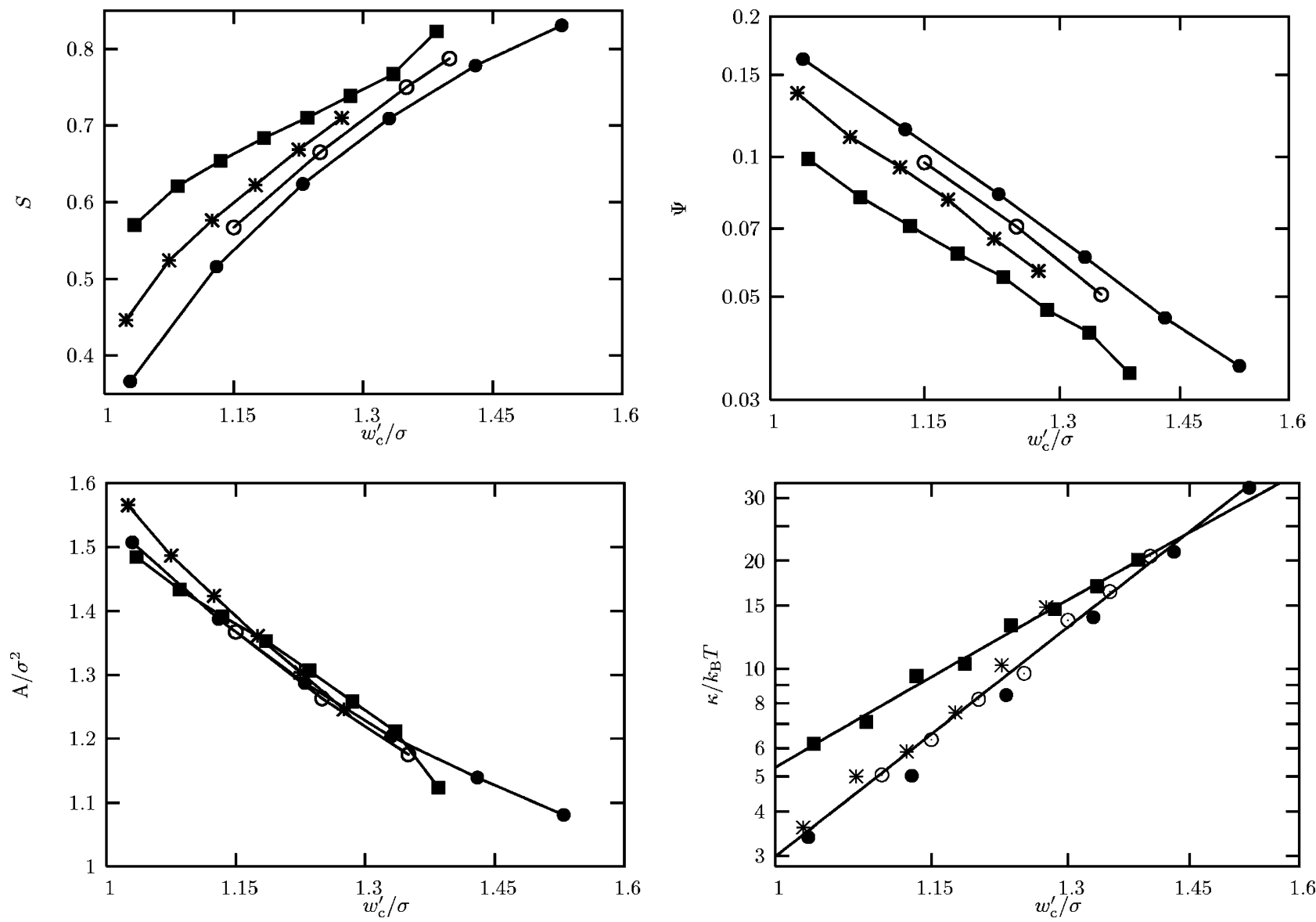

FIG. 7. Basic static properties of the fluid bilayer phase as a function of $w_{c}^{\prime}$ and $k_{\mathrm{B}} T$, where $w_{c}^{\prime}$ indicates a rescaled attractive potential $\left(w_{c}^{\prime}=1+w_{c}-w_{c}^{\mathrm{lg}}\right)$. $w_{c}^{\mathrm{lg}}$ is the value of $w_{c}$ on the liquid-unstable (gas) transition line. Each plot shows four isotherms: $k_{\mathrm{B}} T=0.6 \epsilon$ (filled squares), $k_{\mathrm{B}} T=0.8 \epsilon$ (asterisks), $k_{\mathrm{B}} T=1.0 \epsilon$ (open circles), and $k_{\mathrm{B}} T=1.1 \epsilon$ (filled circles). The values of $w_{c}^{\mathrm{lg}} / \sigma$ for each of these isotherms were $0.815,1.025,1.2$, and 1.27 , respectively. In all cases statistical errors were smaller than the size of the plotted points.

ity density for flipping at time $t$ is given by $r e^{-r t}$, and thus the average time between flip flops is $\langle t\rangle=1 / r$.

\section{B. Constant-temperature cuts}

Figure 7 summarizes the values of orientational order parameter $S$ [Eq. (6)], overlap order parameter $\Psi$ [Eq. (7)], area per lipid $a$, and bending modulus $\kappa$ for constanttemperature scans along the isotherms $k_{\mathrm{B}} T / \epsilon=0.6,0.8,1.0$, and 1.1. In order to more directly compare between the results at different temperatures we applied the rescaling $w_{c}^{\prime}$ $=1+w_{c}-w_{c}^{\mathrm{lg}}$ where $w_{c}^{\mathrm{lg}}$ represents the value of $w_{c}$ at the liquid-gas transition line for each respective temperature (we chose 1 as a reference value rather than 0 to permit the construction of log-scale plots). This allows us to scale out the most obvious effects of temperature and bring the results for all of the isotherms much closer to a common trend.

We first note that the trends for all observables are monotonic, showing a consistent change from bilayers close to the liquid-gas boundary to those close to the liquid-gel boundary. Starting with the structural parameters, $S$ and $\Psi$, we see that both indicate an increase in order with a larger potential range $w_{c}$. This is not immediately obvious because broadening of the attractive potentials could also give the lipids more lateral freedom. Yet, the increase in $S$ indicates that lipids fluctuate less around their average vertical posi- tion, and this goes hand in hand with a concomitant decrease in the overlap $\Psi$ between the vertical density profiles for individual beads. In fact, it turns out that the average bead positions are roughly constant, hence the main mechanism by which $\Psi$ can increase is via the broadening of $\rho(z)$ peaks for individual beads (see Fig. 4). Close to the liquid-gas boundary the bilayer order is rather low. Indeed, visual inspection of bilayers with $\Psi \gtrsim 0.1$ confirms that these are indeed very "fuzzy." Although a clear bilayer structure can still be seen in such cases, we would caution against using these in the attempt to model real lipid systems.

The increase in order becomes more understandable when looking at the average area per lipid. Extending the range of the cohesive potential leads to an overall lateral contraction of the bilayer, thus explaining the reduced fluctuations and thus the behavior of $S$ and $\Psi$. It is remarkable that the area per lipid of all four isotherms agrees after rescaling. This indicates that the lipid density depends purely on the distance from the liquid-gas phase boundary rather than the absolute value of temperature or $w_{c}$ individually.

The area $a$ per lipid can also be used to map the coarsegrained length scale $\sigma$ to experimental lengths. For real phospholipid membranes values around $0.75 \mathrm{~nm}^{2}$ for the area per lipid are typical, ${ }^{32,39}$ while our simulations give values in the range of $1.1-1.5 \sigma^{2}$. Assuming that one coarse- 
grained lipid is equivalent to one real lipid this gives a mapping of roughly $\sigma \simeq 0.7-0.8 \mathrm{~nm}$. An alternative mapping can be obtained by comparing a typical bilayer thickness of roughly $5 \mathrm{~nm}$ with the measured width of the overall lipid density of approximately $5 \sigma$ (see Sec. IV A 2 and Fig. 4), which gives $\sigma \simeq 1 \mathrm{~nm}$. Such good agreement between these two mappings indicates that our very simple three-bead lipids are actually remarkably close to the aspect ratio of real lipids.

The observables presented so far reflect mostly local bilayer properties. In contrast, the bending stiffness is an observable which, even though it ultimately derives from local bilayer properties, yields physics which is accessible by large scale continuum calculations. Much of the theoretical modeling of fluid membranes hinges on the remarkable fact that on sufficiently large length scales they can be described by idealized surfaces with a very simple energy density, for which the bending stiffness $\kappa$ is in almost all cases the only relevant modulus. ${ }^{3,35,36}$ Reproducing experimentally meaningful and easily tunable values for this modulus is therefore one of the key requirements for any coarse-grained membrane modeling that aims at bridging the gap between local and global scales.

Using the procedure described in Sec. IV A 3 we calculated $\kappa$ for each of the four isotherms $k_{\mathrm{B}} T / \epsilon=0.6,0.8,1.0$, 1.1 and for all values of $w_{c}$ in the fluid phase. The two most important conclusions from these data are the following: First, the range of accessible values for the bending stiffness coincides exactly with the experimentally interesting range for usual phospholipids. ${ }^{22}$ Second, the value of $\kappa$ can be easily tuned via one parameter, the potential range $w_{c}$. Beyond that, it is quite remarkable that plotting $\kappa / k_{\mathrm{B}} T$ against the rescaled potential width $w_{c}^{\prime}$ collapses all data points onto one master curve-with the notable exception of $k_{\mathrm{B}} T / \epsilon$ $=0.6$, which also stands out for the diffusion constant (see below). As a guide to the eye, a single line is shown for the three warmest isotherms and a separate line for the coldest one. Just as for the area per lipid it seems thus that the bending modulus depends only on the distance from the liquid-gas boundary. This is plausible since the bending modulus is inversely proportional to the lateral compressibility, which again depends on the lipid density.

Both dynamical properties, the bilayer diffusion constant $D_{b}$ (Fig. 8) and the flip-flop rate $r_{f}$ (Fig. 9), show a clear exponential decay with increasing $w_{c}^{\prime}$. Since we know that for free diffusion $D \propto T$, a simple rescaling by temperature brings the diffusion constants for all isotherms into a rough agreement, with the notable exception of $k_{\mathrm{B}} T / \epsilon=0.6$. We have seen that this same isotherm also gives an anomalous trend in the bilayer stiffness, and one can speculate that this may be due to the unusual shape of the phase diagram (see Fig. 1) between $w_{c} / \sigma=0.8$ and $w_{c} / \sigma=1.1$.

A typical value for the diffusion constant of lipids in real phospholipid membranes is about $1 \mu \mathrm{m}^{2} / \mathrm{s} .{ }^{40}$ Taking an average value of about $0.01 \sigma^{2} / \tau$ from our data, and using the length mapping $\sigma \approx 1 \mathrm{~nm}$ (see above) we obtain a time scale mapping of $\tau \approx 10 \mathrm{~ns}$. Although we do not place great quantitative weight on such an approximate calculation, it does serve to illustrate that the time scales accessible by us are

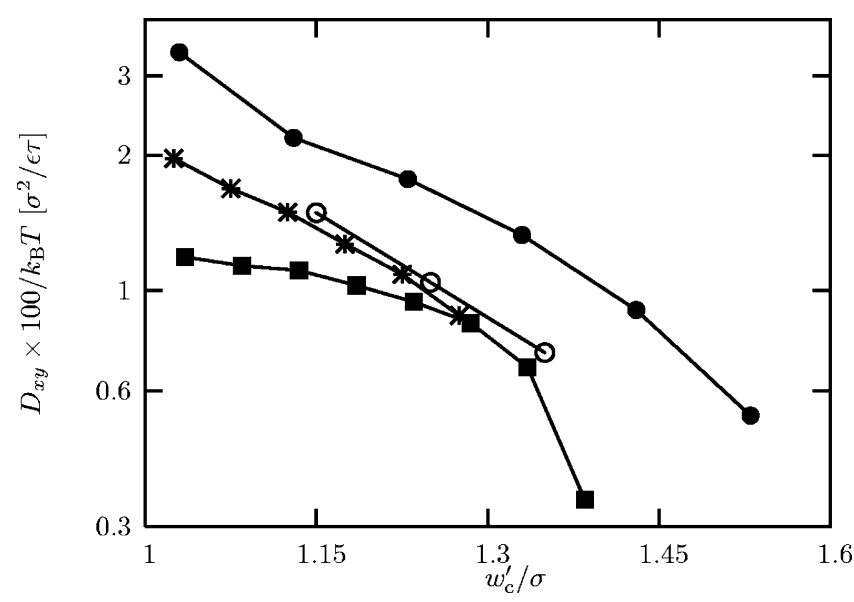

FIG. 8. Diffusion constant as a function of rescaled potential width $w_{c}^{\prime}=1$ $+w_{c}-w_{c}^{\mathrm{lg}}$. The symbols and shifts $w_{c}^{\mathrm{lg}}$ are the same as in Fig. 7.

extremely long, being of the order of milliseconds. This is long enough, for example, to allow vesicles to self-assemble or fuse, and for macroscopic phase separation to take place.

In the case of flip-flop rates we found a strong dependence on temperature which is in accordance with the fact that this is a thermally activated process. The most important point to note, however, is that the range of actual values for $r_{f}$ obtained by us are many orders of magnitude faster than those typically found for artificial phospholipid bilayers in experiments. ${ }^{41,42}$ Such a large discrepancy is not as alarming as it may at first seem since the rate $r_{f}$ is exponentially dependent on the activation energy for flip $E_{f}$ (Arrhenius law). We can therefore account for a very large difference in $r_{f}$ by a relatively small discrepancy in $E_{f}$. Nevertheless, it is clear that the lipids in our model undergo flip flop too easily compared with real lipids. If one were specifically interested in this aspect of the dynamics, this would clearly represent a problem; however, in many other cases it provides an advantage because the system will approach equilibrium more rapidly. If an accurate flip-flop rate is important we anticipate that our model lipids could be made to flip much less readily simply by increasing the chain length slightly (e.g., four bead lipids) and imposing a much stronger head-tail repulsion.

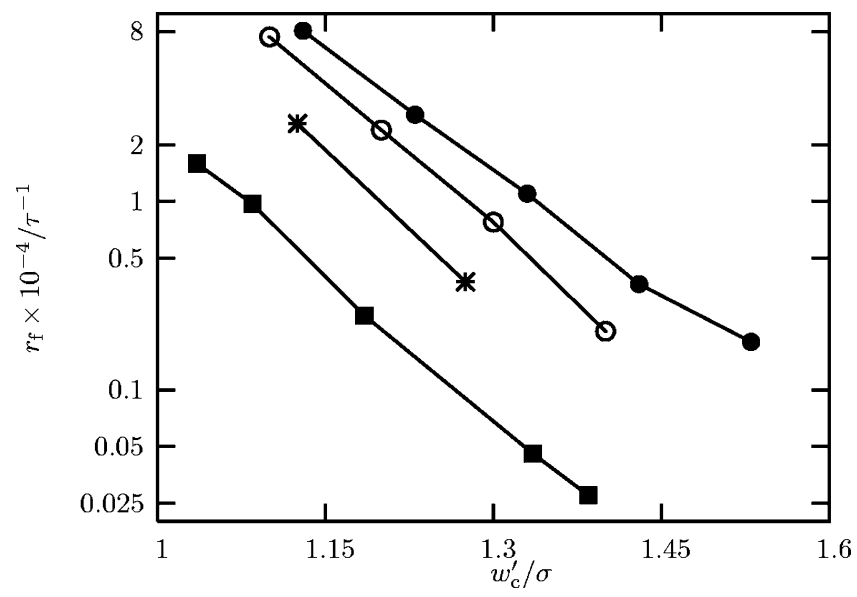

FIG. 9. Flip-flop rate $r$ as a function of rescaled potential width $w_{c}^{\prime}=1$ $+w_{c}-w_{c}^{\mathrm{lg}}$. The symbols and shifts $w_{c}^{\mathrm{lg}}$ are the same as in Fig. 7. 


\section{Constant $w_{c}$ profiles: Gel-fluid transition}

At constant values of $w_{c}$ one can vary the temperature to observe both liquid-gel and liquid-gas transitions. Since we studied lipid phase behavior at vanishing lateral tension, the liquid-gas boundary is necessarily sharp in our case. However, it is worth mentioning that under constant volume conditions alternative low-density phases such as spherical or cylindrical micelles can be observed. Using the present model we have, in fact, observed such micellar phases, and together with one more control parameter-overall lipid density - their phase behavior could be studied as well. This, however, is not the purpose of our present work and will be presented elsewhere. Here we concentrate on the transition between liquid and gel phases at vanishing lateral tension. There are several different ordered phases which collectively can be referred to as gels, ${ }^{34,43}$ but we shall not attempt to identify all of these. Indeed, our rather simple three-bead model was neither designed to reproduce such subtleties of lipid ordering nor would we actually expect to observe the full zoo of ordered bilayer phases. Nevertheless, it is worthwhile to address one particular issue in this regard which pertains to our choice of a single chain rather than a doublechain lipid. A number of previous simulation studies ${ }^{44,45}$ have examined phase diagrams for coarse-grained singleand double-chain lipids in the liquid-gel region as a function of temperature and head group size. For single chains the interdigitated gel phase $L_{\beta I}$ and nontilted gel phase $L_{\beta}$ were observed, but only with two-tailed lipids could one observe the tilted gel phase $L_{\beta^{\prime}}$. Such observations naturally lead to questions about the validity of coarse-grained models where single-rather than double-tailed surfactant molecules are used. Our expectation, however, is that much of the unusual behavior observed for the single-chain lipids in Refs. 44 and 45 can be attributed to their high aspect ratio (at least 6:1 compared with real lipids, $3, \ldots, 4: 1$ ), combined with the soft repulsions used. These facts would naturally lead to a strong tendency to form the $L_{\beta I}$ phase, possibly at the expense of the $L_{\beta^{\prime}}$ phase. Since our model is based on a single-chain surfactant, one might suppose that it should suffer from the same problems. In fact, this is not the case for two reasons: (a) our lipid molecules are close to the natural aspect ratio $(3, \ldots, 4: 1$, though perhaps slightly short) and (b) our repulsive potentials are of relatively hard Lennard-Jones type. Thus the tendency for interdigitation in our case is very low (see Sec. IV A 2) and our lipids bear closer resemblance to the two-tailed surfactants used in other studies.

In Fig. 10 we show the variation of lipid area $a$ with temperature for three values of $w_{c}$. In all cases we chose to employ continuous temperature scans in order to allow the barostat to smoothly follow the substantial changes in box size involved. The important question then arises: "Is our rate of cooling slow enough?" We checked this by comparing plots of $a$ vs $T$ for runs with four different cooling rates: $10^{-4}, 10^{-5}, 2.5 \times 10^{-6}$, and $10^{-6}$ (in units of $\epsilon / k_{\mathrm{B}} \tau$ ), from which one can see a clear deviation for the fastest rate but qualitatively very similar results for all slower ones. The only difference is that slightly sharper transition boundaries can be seen as the rate is lowered, even among the slowest
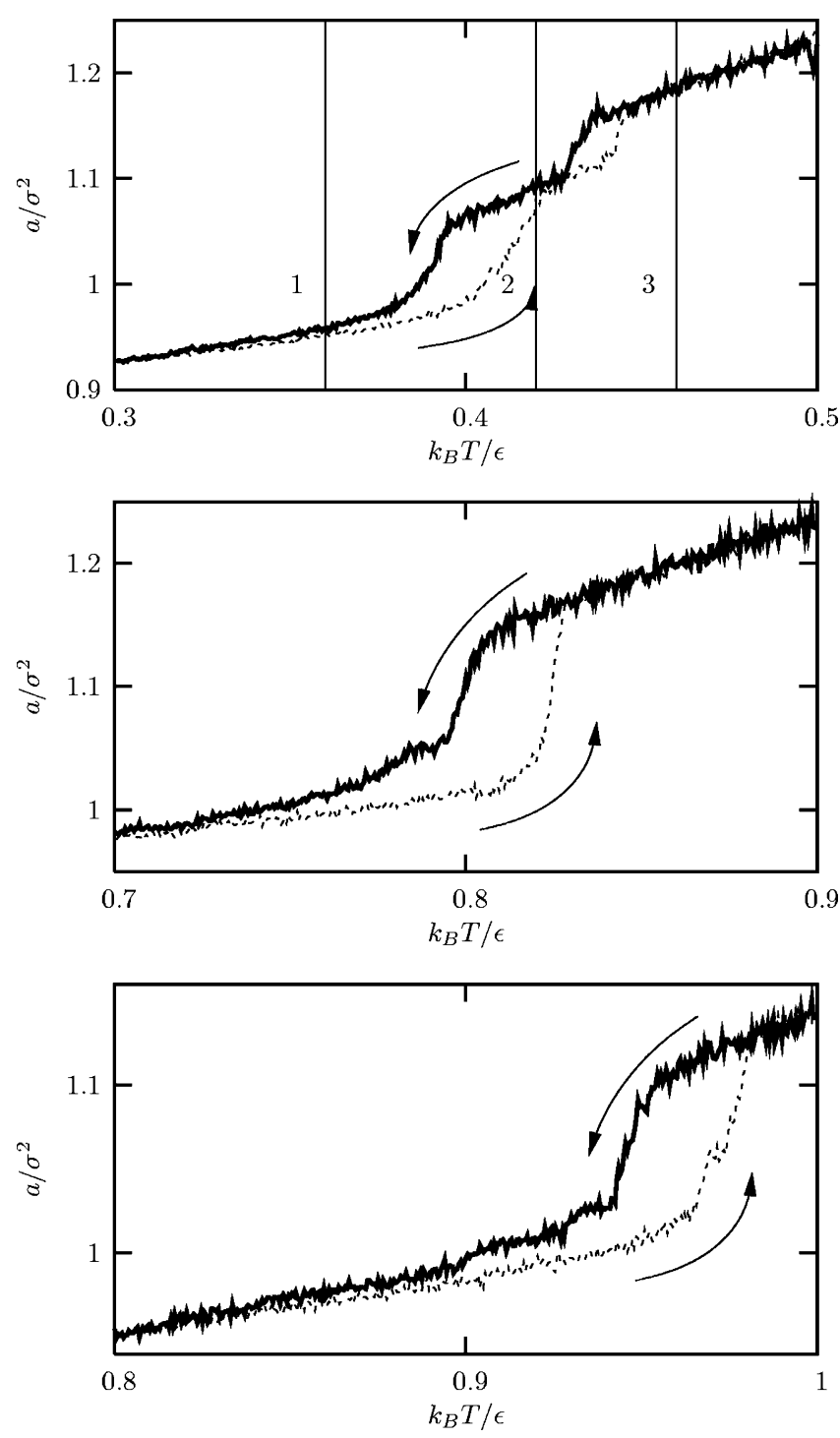

FIG. 10. Variation of the area per lipid $a$ across the gel-fluid-phase boundary. Each figure shows a cooling-heating hysteresis for a particular value of $w_{c}$. From top to bottom the values of $w_{c} / \sigma$ used were 1.0, 1.4, and 1.6. The arrows indicate the direction of temperature change. The rate of temperature change was $2.5 \times 10^{-6} \epsilon / k_{\mathrm{B}} \tau$ for the top plot and $5 \times 10^{-6} \epsilon / k_{\mathrm{B}} \tau$ for the bottom two plots. The three vertical lines in the uppermost plot indicate the temperatures where the order parameter of Fig. 11 has been measured.

three rates. As a compromise between speed and resolution we chose the intermediate rate $5 \times 10^{-6} \epsilon / k_{\mathrm{B}} \tau$ for runs with $w_{c} / \sigma=1.4$ and $w_{c} / \sigma=1.6$ which display only a single transition. For runs with $w_{c} / \sigma=1.0$, where two transitions must be resolved, we chose the slower rate, $2.5 \times 10^{-6} \epsilon / k_{\mathrm{B}} \tau$.

Before focusing on the results at each value of $w_{c}$ it is interesting to note that all runs show quite a strong hysteresis across the transition boundary as is typical of first-order transitions. However, there is also a long tail to the hysteresis which appears during cooling. This is most likely due to the fact that the kinetics during gelling is strongly determined by the slow healing of defects. Indeed, we often observed such defects, many of which could be seen to dissappear during further slow cooling.

Turning now to the results at $w_{c}=1.0$ we find that there are two clear and sudden transitions during cooling as well as 


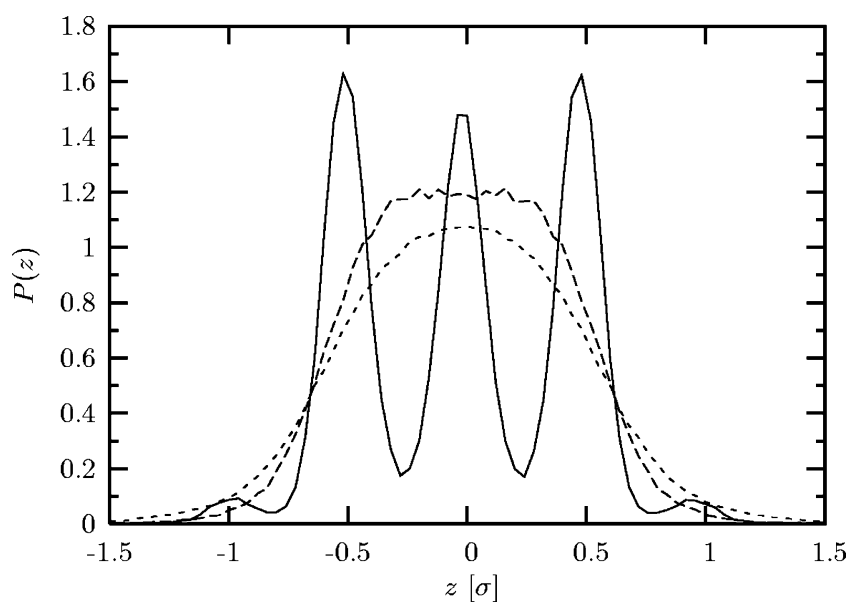

FIG. 11. Probability density $\rho(z)$ of the height difference $z$ between a lipid and its six immediate neighbors. The solid, dashed, and dotted curves correspond to the temperatures indicated by lines 1, 2, and 3 in Fig. 10, respectively.

the reverse for heating. During cooling, both transitions involve a contraction of the area $a$ and an increase in the orientational order $S$ (not shown); however, if we look at the diffusion constant $D_{b}$ we find that it decreases suddenly from about $2 \times 10^{-3} \sigma^{2} / \tau$ to approximately $4 \times 10^{-5} \sigma^{2} / \tau$ in the first (higher temperature) transition but does not decrease further during the second (lower temperature) transition. In fact, the very small value of the diffusion constant is numerically hard to determine accurately, but the overall drop by about two orders of magnitude is probably robust and corresponds well to what is known for typical phospholipid bilayers. ${ }^{40}$ Thus we have a first transition from fluid to gel and a second transition at a lower temperature to a different gel phase. In order to understand what is actually occurring in the gel-gel transition we require a suitable order parameter, and it turns out that local lipid packing is what one has to look at. We calculated a histogram of height differences $\Delta z$ between a lipid head and that of its six nearest neighbors. The results are shown in Fig. 11 for bilayers at temperatures $k_{\mathrm{B}} T / \epsilon=0.38,0.42$, and 0.46 corresponding to each of the three phases. Both the fluid phase and the high-temperature gel phase show a distribution peaked about $\Delta z=0$; however, there is a striking change as we go to the phase at $k_{\mathrm{B}} T / \epsilon$ $=0.38$ which shows three peaks at $\Delta z= \pm 0.5 \sigma$ and 0 . This shows that the lipids are now packed in an off-centered manner rather like oranges in a crate. Puzzlingly, however, an equal proportion of lipids is found at $\Delta z=0$ which would not occur if a perfect packing was obtained. This might be due to the presence of a still large number of defects.

At larger values of $w_{c}$ there is just a single transition from the fluid to the gel phase. This gel phase is packed similarly to the low-temperature gel phase for $w_{c}=1.0$ since it also exhibits a three-peak structure in the histogram of $\Delta z$.

Finally, the plots of area per lipid versus temperature also contain information besides the evident phase transitions. Their slope yields the value of the lateral thermal area expansivity, defined by

$$
\alpha_{T}=\frac{1}{\langle a\rangle} \frac{\partial\langle a\rangle}{\partial T} .
$$

For instance, the system with $w_{c} / \sigma=1.6$ has a slope $\partial\langle a\rangle / \partial T \approx 0.766 \sigma^{2} /\left(\epsilon / k_{\mathrm{B}}\right)$ in the fluid phase and an area per lipid of $1.142 \sigma^{2}$ at $k_{\mathrm{B}} T / \epsilon=1$. Identifying this temperature with room temperature, we obtain a thermal expansivity of $\alpha_{T} \approx 2.2 \times 10^{-3} \mathrm{~K}^{-1}$, which coincides remarkably well with the value measured by Kwok and Evans for fluid lecithin bilayers $\left(2.4 \times 10^{-3} \mathrm{~K}^{-1}\right){ }^{46}$

\section{MEMBRANE STRETCHING AND PORE OPENING}

So far we have studied membranes under zero lateral tension. If we now apply extensional stress, the area per lipid will increase, up to the point where structural stability of the bilayer is breached. Beyond a critical stress a pore can be nucleated, which then grows indefinitely, i.e., the bilayer ruptures. This scenario has been described theoretically by Litster. ${ }^{47}$ Important bilayer properties (e.g., the line tension of an open edge) could be extracted from observables such as the critical stress or the critical pore size, but it is evidently experimentally very difficult to study membranes at the brink of rupture. However, in a simulation it is very easy to perform measurements in a different ensemble, namely, one of the constant area of the entire bilayer. Beyond some critical strain one would now expect a pore to open, but since this now relieves much of the stress, pore growth stops and one obtains a stable pore of well-defined size. Assuming a harmonic extensibility of the bilayer itself, as well as a constant line tension at a pore rim, Farago ${ }^{19}$ and Tolpekina et al. ${ }^{26}$ gave a simple theoretical model which relates the resulting pore size as well as the stress-strain relation to key bilayer properties such as the extensibility and the line tension. We summarize the key results in Appendix B.

Using our simulation model with the parameters $w_{c} / \sigma$ $=1.6$ and $k_{\mathrm{B}} T / \epsilon=1.1$ we determined the equilibrium lateral tension $\Sigma$ as a function of total box area $\left(A=L_{x} \times L_{y}\right)$. For each value of the area this was done by placing 4000 lipids in a bilayer configuration spanning the $x y$ plane of the simulation box. After allowing for an equilibration time of $3 \times 10^{4} \tau$ we then simulated for a further $3 \times 10^{4} \tau$, during which time the lateral tension $\Sigma=-L_{z}\left(p_{x x}+p_{y y}\right) / 2$ was measured. The resulting plot of $A$ vs $\Sigma$ is shown in Fig. 12. Three main regimes are clearly distinguishable, separated by two values of the bilayer area. First, at a particular area $A_{0}$ the tension vanishes. Boxes with a smaller area yield a negative tension, i.e., a positive pressure. The bilayer is under compressional strain, which it evades very soon by buckling. Conversely, boxes with an area larger than $A_{0}$ subject the bilayer to extensional strain and create a proportional increase in tension. At some particular value for the bilayer area the energy stored in the extension, which grows quadratically with the strain, must exceed that of a bilayer with a pore, since the pore size cannot grow faster than the strain, and the line tension is proportional to the square root of pore size. Once the pore opens it releases much of the stress and further expansion of the area remarkably leads to a further decrease in the stress. This "wrong" sign for the extensibility 


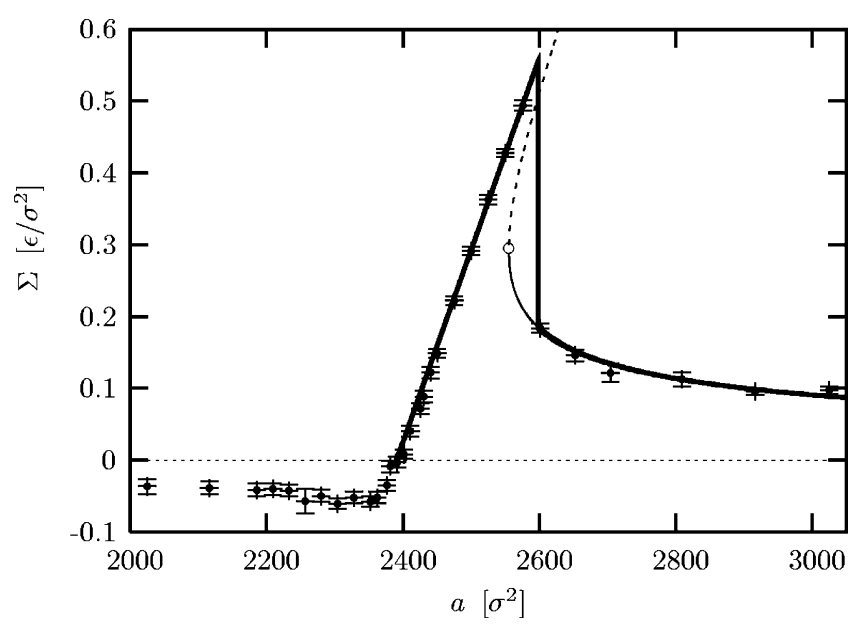

FIG. 12. Bilayer tension $\Sigma$ as a function of (projected) area $A$ for a flat membrane sheet with $w_{c} / \sigma=1.6$ at $k_{\mathrm{B}} T / \epsilon=1.1$. The bold solid line is a fit to the model of Farago (Ref. 19) and Tolpekina et al. (Ref. 26) [see also Eq. (B5) in Appendix B]; the fine solid and dashed curves indicate the metastable and unstable branches, respectively.

explains why a stable pore cannot be achieved under constant tension conditions.

The model of Farago ${ }^{19}$ and Tolpekina et al. $^{26}$ gives perfect agreement with the measured data ${ }^{48}$ (see lines in Fig. 12). This, however, not only shows that their theoretical assumptions were correct but also provides a reference for comparison of our model with the well-known model of Goetz and Lipowsky ${ }^{49}$ and Goetz et al. ${ }^{50}$ since Tolpekina et al. ${ }^{26}$ successfully fitted their results to this earlier model. Contrary to ours, the model of Goetz and Lipowsky and Goetz et al. includes explicit solvent. That both simulations can be described very well by the same theory implies that their physical behavior under extensional stress is indeed very similar.

Fitting the linear extensional regime of our simulational data to the stretching model we obtain a modulus of $M \approx 6.4 \epsilon / \sigma^{2}$. Translating this to real values depends again on the mapping. Using $1.1 \epsilon=k_{\mathrm{B}} T=4.1 \mathrm{pN} \mathrm{nm}$ as well as a length mapping of $\sigma \approx 0.9 \mathrm{~nm}$ gives $M \approx 30 \mathrm{mN} / \mathrm{m}$. This is at the lower end of what one typically expects for phospholipid bilayers. ${ }^{21,23,46}$ The rupture tension is $\Sigma_{\text {pore }} \approx 0.55 \epsilon / \sigma^{2}$, which translates to about $2.5 \mathrm{mN} / \mathrm{m}$, while the line tension is $\gamma \approx 1.2 \epsilon / \sigma$, giving about $5 \mathrm{pN}$. Both these values are very reasonable, ${ }^{51,52}$ but one has to be careful, since they depend in a nontrivial way on the system size. ${ }^{26}$

\section{CONCLUSIONS}

We have presented a model for the solvent-free simulation of coarse-grained lipid bilayer membranes that is free of the major problems encountered by earlier efforts towards this goal. It robustly self-assembles to a fluid phase, uses simple two-body potentials, and is highly tunable. We have emphasized that we regard its functioning to rely on a general principle: the presence of sufficiently broad tail attraction potentials. This gives lipids a chance of lateral mobility while maintaining fluidity. Since rearrangements and their concomitant local increase in pair distances do not immediately cost most of the binding energy, the entropy gain upon fluidization is not inhibited energetically. In this context we remind the reader that it is a well-known fact from colloidal physics that if the range of attraction is too short compared to the particle's hard-core radius, no more fluid phase exists. ${ }^{53-55}$ This principle should therefore also help if extensions to our present model, such as more beads per lipid or a restriction of the long-ranged attraction to specific beads only, are to be employed.

We have measured many physical characteristics of our membrane model and have illustrated that they are easily tunable in a controlled way. Often their values fall well inside or close to the experimentally interesting range without any explicit careful tuning. This indicates that the model is clearly flexible enough and can serve as a good starting point for quantitative matching to specific systems in the spirit of systematic coarse graining, which is an approach that has only recently been employed for lipid membrane simulations $^{31,56}$ but is well established in other fields of soft matter. ${ }^{57,58}$ Since we have demonstrated that we can readily achieve mesoscopic length scales beyond $100 \mathrm{~nm}$ and time scales of milliseconds, this opens up a wide range of interesting mesoscale problems that can with some additional parameter matching be simulated quantitatively. Efforts in this direction are currently under way.

\section{ACKNOWLEDGMENTS}

We thank Oded Farago, Friederike Schmid, Hiroshi Noguchi, Gregoria Illya, Kurt Kremer, and Bernward Mann for valuable discussions. We also gratefully acknowledge financial support by the fGerman Science Foundation under Grant De775/1-2.

\section{APPENDIX A: SPECTRAL DAMPING}

In Sec. IV A 3 we determined the bending modulus from the membrane fluctuation spectrum, which in turn was extracted from the simulation by interpolating the lipid positions onto a $16 \times 16$ grid. However, any method which interpolates continuous variables onto grid points is prone to discretization artifacts, and it is crucial to identify them in advance.

We illustrate the difficulty in the one-dimensional case first. Let $h_{q}(x)$ be a single mode on the linear interval $[0 ; L]$, given by

$$
h_{q}(x)=e^{i\left(q x+\varphi_{q}\right)}, \quad q=\frac{2 \pi n}{L}, \quad n \in \mathbb{Z},
$$

where $\varphi_{q}$ is a $q$-dependent phase. On the grid this mode has the values $H_{q}(k)=h_{q}\left(x_{k}\right)$ with $x_{k}=k L / N$ and $k \in\{0,1, \ldots, N$ $-1\}$. The inverse finite Fourier transform $\hat{H}_{q}(u)$ of these sample points is given by

$$
\begin{aligned}
\hat{H}_{q}(u) & =\frac{1}{N} \sum_{k=-N / 2}^{(N / 2)-1} H_{q}(k) e^{-2 \pi i u k / N} \\
& =\frac{1}{N} \sum_{k=-N / 2}^{(N / 2)-1} e^{2 \pi i(n-u) k / N} e^{i \varphi_{k}}=e^{i \varphi_{k}} \delta_{n, u} .
\end{aligned}
$$

This is expected: If $n=u$ we get back the phase of our mode, 
but if $n \neq u$ we get 0 , reflecting the fact that the wave numbers $u$ and $n$ do not match.

The situation changes once we go beyond plain sampling. In the bilayer situation under study our mode $h_{q}(x)$ is being represented by many off-lattice points $x \in[0 ; L]$. Grid interpolation was achieved by assigning to any grid point the average of the $h_{q}(x)$ for the $x$ values closest to that grid point. If there are many such points, this basically means that we do not sample the mode at the grid point, but rather sample its average around that grid point. Let us thus define the average-sampled interpolation function $\bar{H}_{q}(k)$ via

$$
\begin{aligned}
\bar{H}_{q}(k) & =\frac{1}{L / N} \int_{x_{k}-(L / 2 N)}^{x_{k}+(L / 2 N)} d x e^{i\left(q x+\varphi_{q}\right)} \\
& =\frac{N}{i q L} e^{i\left(q k L / N+\varphi_{q}\right)}\left(e^{i q L / 2 N}-e^{-i q L / 2 N}\right) \\
& =\operatorname{sinc}\left(\frac{n}{N}\right) H_{q}(k),
\end{aligned}
$$

where we introduced $\operatorname{sinc}(x)=\sin (\pi x) / \pi x$. Evidently $\bar{H}_{q}(k)$ differs from the ordinary sampled interpolation function $H_{q}(k)$ by an additional wave-vector-dependent but positionindependent prefactor. This factor goes to zero at $n=N$, which implies that such modes are entirely averaged away, but already at the boundary of the Brillouin zone, at $n=N / 2$, it has the disconcertingly small value $\operatorname{sinc} \frac{1}{2}=2 / \pi$ $\approx 0.64$. Once the mode has arrived on the mesh, its amplitude is no longer what it used to be off lattice.

The inverse finite Fourier transform of $\bar{H}_{q}(k)$ is also multiplied by this prefactor,

$$
\begin{aligned}
\hat{\bar{H}}_{q}(u) & =\frac{1}{N_{k=-(N / 2)}} \sum^{N / 2-1} \operatorname{sinc}\left(\frac{n}{N}\right) H_{q}(k) e^{-2 \pi i u k / N} \\
& =\operatorname{sinc}\left(\frac{n}{N}\right) \hat{H}_{q}(u),
\end{aligned}
$$

and thus the power spectrum is damped according to

$$
\left|\hat{\bar{H}}_{q}(u)\right|^{2}=\operatorname{sinc}^{2}\left(\frac{n}{N}\right)\left|\hat{H}_{q}(u)\right|^{2}=\operatorname{sinc}^{2}\left(\frac{n}{N}\right) \delta_{n, u} .
$$

At the boundary of the Brillouin zone this factor is $(2 / \pi)^{2}$ $\approx 0.41$ and thus not at all negligible. Notice that this does not depend on the mode amplitude. In other words, even if the mode is only slightly excited and the membrane thus looks benignly flat, the estimate for the power spectrum derived from the gridded function is systematically too low.

It is easy to see that in two dimensions this result generalizes to

$$
\left|\hat{\bar{H}}_{\boldsymbol{q}}(\mathbf{u})\right|^{2}=\operatorname{sinc}^{2}\left(\frac{n_{x}}{N}\right) \operatorname{sinc}^{2}\left(\frac{n_{y}}{N}\right) \delta_{\mathbf{n}, \mathbf{u}} .
$$

The fact that this damping is $\boldsymbol{q}$ dependent renders it potentially harmful. Figure 5 shows that a typical zero-tension fluctuation spectrum at low values decays as $q^{-4}$ but starts to bend upwards once the wave vector approaches microscopic scales. The spectral damping discussed above will act to suppress this upturn and delay it to even larger $q$ values. The $q^{-4}$ regime might therefore appear to extend further than it actually does, and one might thus be tempted to fit an expression valid only in the bending regime to data which owe their exponent to a combination of, say, protrusion modes and spectral damping artifacts.

In our mode analysis we avoided such artifacts by simply dividing out the damping factor.

\section{APPENDIX B: MEMBRANE PORE IN A CONSTANT- AREA ENSEMBLE}

For the total energy of an elastic bilayer spanned inside a frame of area $A$ which displays a harmonic extensibility with modulus $M$ and which has a line tension $\gamma$, Refs. 19 and 26 write

$$
E=\frac{1}{2} M \frac{\left(A-A_{0}-\pi R^{2}\right)^{2}}{A_{0}}+2 \pi \gamma R,
$$

where $A_{0}$ is the tensionless area of that bilayer and $R$ the radius of a circular pore. If $R$ is indeed nonzero, its value has to be chosen such as to minimize $E$.

It is useful to rescale variables. Let us define a characteristic length scale $\lambda$ and with its help introduce a dimensionless pore radius and area extension,

$$
\lambda^{3}=\frac{\gamma A_{0}}{\pi M}, \quad \tilde{R}=\frac{R}{\lambda}, \quad \text { and } B=\frac{A-A_{0}}{\pi \lambda^{2}} .
$$

The equation for the optimal pore radius, $\partial E / \partial R=0$, then reduces to

$$
\widetilde{R}^{3}-B \widetilde{R}+1=0 .
$$

Hence, the pore opening scenario will exclusively depend on only one characteristic dimensionless variable, $B$; furthermore, all length scales will be proportional to $\lambda$ with a prefactor that depends on $B$ alone.

It is easy to see that $R=0$ is always a local minimum for $E$ (even though the derivative does not vanish there). For $B>B_{c}=3 / 2^{2 / 3} \approx 1.89$ two more stationary radii appear as solutions of Eq. (B3):

$$
\tilde{R}_{ \pm}(B)=2 \sqrt{B / 3} \cos \frac{\pi \pm \arctan \sqrt{4(B / 3)^{3}-1}}{3} .
$$

The solution $\widetilde{R}_{-}(B)$ corresponds to a local minimum and for large $B$ asymptotically scales like $\sqrt{B}$; for $B>B_{\text {pore }}=3 / 2^{1 / 3}$ $\approx 2.38$ this minimum becomes the global one and a discontinuous transition to a pore state occurs, which displays a system size-dependent energy barrier of $E_{\text {barrier }} \approx 1.38 \gamma \lambda$ $\approx 0.94 \gamma^{4 / 3} M^{-1 / 3} A_{0}^{1 / 3}$. In this pore state the tension is given by

$$
\Sigma_{-}(B)=\frac{\gamma}{\lambda}\left[B-\widetilde{R}_{-}^{2}(B)\right] \simeq \frac{B \gg 1}{\sqrt{B}}+\mathcal{O}\left(B^{-2}\right) .
$$

At the transition point the tension drops exactly by a factor 3 , and the system size-dependent rupture tension is given by $\Sigma_{\text {pore }}=3 / 2^{1 / 3} \gamma / \lambda \approx 2.38 \gamma / \lambda \approx 3.49 \gamma^{2 / 3} M^{1 / 3} A_{0}^{-1 / 3}$.

${ }^{1}$ D. Zahn and J. Brickmann, Phys. Chem. Chem. Phys. 3, 848 (2001).

${ }^{2}$ A. M. Smondyrev and G. A. Voth, Biophys. J. 82, 1460 (2002).

${ }^{3}$ U. Seifert, Adv. Phys. 46, 13 (1997). 
${ }^{4}$ P. Kumar, G. Gompper, and R. Lipowsky, Phys. Rev. Lett. 86, 3911 (2001).

${ }^{5}$ H. Noguchi and G. Gompper, Phys. Rev. Lett. 93, 258102 (2004).

${ }^{6}$ J. C. Shillcock and R. Lipowsky, Nat. Mater. 4, 225 (2005).

${ }^{7}$ H. Noguchi and M. Takasu, Phys. Rev. E 64, 041913 (2001).

${ }^{8}$ M. Müller, K. Katsov, and M. Schick, Biophys. J. 85, 1611 (2003).

${ }^{9}$ S. L. Veatch and S. L. Keller, Phys. Rev. Lett. 89, 268101 (2002).

${ }^{10}$ S. L. Veatch and S. L. Keller, Biophys. J. 85, 3074 (2003).

${ }^{11}$ T. Baumgart, S. T. Hess, and W. W. Webb, Nature (London) 425, 821 (2003).

${ }^{12}$ M. Laradji and P. B. Sunil Kumar, Phys. Rev. Lett. 93, 198105 (2004).

${ }^{13}$ H. Noguchi and M. Takasu, Biophys. J. 83, 299 (2002).

${ }^{14}$ J.-M. Drouffe, A. C. Maggs, and S. Leibler, Science 254, 1353 (1991).

${ }^{15}$ S. J. Marrink, A. H. de Vries, and A. E. Mark, J. Phys. Chem. B 108, 750 (2004).

${ }^{16}$ H. Noguchi and M. Takasu, J. Chem. Phys. 115, 9547 (2001).

${ }^{17}$ H. Noguchi and M. Takasu, Phys. Rev. E 65, 051907 (2002).

${ }^{18}$ G. Brannigan and F. L. H. Brown, J. Chem. Phys. 120, 1059 (2004).

${ }^{19}$ O. Farago, J. Chem. Phys. 119, 596 (2003).

${ }^{20}$ G. Brannigan, L. C.-L. Lin, and F. Brown, Eur. Biophys. J. (in press).

${ }^{21}$ E. Evans and W. Rawicz, Phys. Rev. Lett. 64, 2094 (1990).

${ }^{22}$ U. Seifert and R. Lipowsky, Handbook of Biological Physics (Elsevier, Amsterdam, 1995), Vol. 1A.

${ }^{23}$ W. Rawicz, K. C. Olbrich, T. McIntosh, D. Needham, and E. Evans, Biophys. J. 79, 328 (2000).

${ }^{24}$ I. R. Cooke, K. Kremer, and M. Deserno, Phys. Rev. E 72, 011506 (2005).

${ }^{25}$ G. Brannigan, P. F. Philips, and F. L. H. Brown, Phys. Rev. E 72, 011915 (2005).

${ }^{26}$ T. V. Tolpekina, W. K. den Otter, and W. J. Briels, J. Chem. Phys. 121, 8014 (2004)

${ }^{27}$ G. S. Grest and K. Kremer, Phys. Rev. A 33, 3628 (1986).

${ }^{28}$ If beads have a mass $m$, the $\mathrm{LJ}$ time scale is given by $\tau=\sigma \sqrt{m / \epsilon}$.

${ }^{29}$ A. Kolb and B. Dünweg, J. Chem. Phys. 111, 4453 (1999).

${ }^{30} \mathrm{http}: / /$ www.espresso.mpg.de

${ }^{31}$ J. C. Shelley, M. Y. Shelley, R. C. Reeder, S. Bandyopadhyay, and M. Klein, J. Phys. Chem. B 105, 4464 (2001).

${ }^{32}$ T. Husslein, D. M. Newns, P. C. Pattnaik, Q. Zhong, P. B. Moore, and M. L. Klein, J. Chem. Phys. 109, 2826 (1998).

${ }^{33}$ A. H. de Vries, S. Yefimov, A. E. Mark, and S. J. Marrink, Proc. Natl. Acad. Sci. U.S.A. 102, 5392 (2005).
${ }^{34}$ M. Kranenburg and B. Smit, J. Phys. Chem. B 109, 6553 (2005).

${ }^{35}$ P. B. Canham, J. Theor. Biol. 26, 61 (1970).

${ }^{36}$ W. Helfrich, Z. Naturforsch. C 28, 693 (1973).

${ }^{37}$ R. Lipowsky and S. Grotehans, Europhys. Lett. 23, 599 (1993).

${ }^{38}$ Just as in a typical experiment we measure the effective diffusion in the projected plane, which is not the same as the bare diffusion in the bilayer surface because of membrane undulations. For a discussion see Ref. 59.

${ }^{39}$ W. Shinoda, N. Namiki, and S. Okazaki, J. Chem. Phys. 106, 5731 (1997).

${ }^{40}$ P. F. Fahey and W. W. Webb, Biochemistry 17, 3046 (1978).

${ }^{41}$ J. Liu and J. C. Conboy, Biophys. J. 89, 2522 (2005).

${ }^{42}$ M. A. Kol, A. I. P. M. de Kroon, D. T. S. Rijkers, A. J. Killian, and B. de Kruijff, Biochemistry 40, 10500 (2001).

${ }^{43}$ Handbook of Biological Physics, edited by R. Lipowsky and E. Sackmann vol. 1+2 (Elsevier, Amsterdam, 1995).

${ }^{44}$ M. Kranenburg, M. Venturoli, and B. Smit, J. Phys. Chem. B 107, 11491 (2003).

${ }^{45}$ M. Kranenburg, M. Venturoli, and B. Smit, Phys. Rev. E 67, 060901 (2003).

${ }^{46}$ R. Kwok and E. Evans, Biophys. J. 35, 637 (1981).

${ }^{47}$ J. D. Litster, Phys. Lett. 53A, 193 (1975).

${ }^{48}$ There are three fitting parameters; zero tension area $A_{0}$, stretching modulus $M$, and line tension $\gamma$ (see Appendix B). These can be obtained purely from the initial stretching regime and the point of rupture. Notably, the subsequent drop in tension and the shape of the curve after pore opening are then completely determined and not subject to further fitting.

${ }^{49}$ R. Goetz and R. Lipowsky, J. Chem. Phys. 108, 7397 (1998).

${ }^{50}$ R. Goetz, G. Gompper, and R. Lipowsky, Phys. Rev. Lett. 82, 221 (1999).

${ }^{51}$ E. Evans, V. Heinrich, F. Ludwig, and W. Rawicz, Biophys. J. 85, 2342 (2003).

${ }^{52}$ S. Loi, G. Sun, V. Franz, and H. J. Butt, Phys. Rev. E 66, 031602 (2002).

${ }^{53}$ A. P. Gast, C. K. Hall, and W. B. Russel, J. Colloid Interface Sci. 96, 251 (1983).

${ }^{54}$ M. H. J. Hagen and D. Frenkel, J. Chem. Phys. 101, 4093 (1994).

${ }^{55}$ A. A. Louis, Philos. Trans. R. Soc. London, Ser. A 359, 939 (2001).

${ }^{56}$ S. Izvekov and G. A. Voth, J. Phys. Chem. B 109, 2469 (2005).

${ }^{57}$ F. Müller-Plathe, ChemPhysChem 3, 754 (2002).

${ }^{58}$ M. Praprotnik, L. Delle Site, and K. Kremer, J. Chem. Phys. (in press).

${ }^{59}$ E. Reister and U. Seifert, Europhys. Lett. 71, 859 (2005). 